\title{
General Constrained Deformations Based on Generalized Metaballs
}

\author{
Xiaogang Jin ${ }^{\natural}$, Y. F. $\mathrm{Li}^{\dagger}$ \& Qunsheng Peng ${ }^{\text {के }}$ \\ ${ }^{i}$ State Key Lab of CAD\&CG, Zhejiang University, Hangzhou, 310027 \\ E-mail:zucad@public.hz.z.j.cn \\ ${ }^{\dagger}$ Department of Manufacturing Engineering and Engineering Management, \\ City University of Hong Kong, 83 Tat Chee Avenue, Kowloon, HongKong
}

\begin{abstract}
Space deformation is an important tool in computer animation and shape design. In this paper we propose a new local deformation model based on generalized metaballs. The user specifies a series of constraints, which can be made up of points, lines, surfaces and volumes, their effective radii and maximum displacements, the deformation model creates a generalized metaball for each constraint. Each generalized metaball is associated with a potential function centered on the constraint, the potential function drops from 1 on the constraint to 0 on the effective radius. This deformation model operates on the local space and is independent of the underlining representation of the object to be deformed. The deformation can be finely controlled by adjusting the parameters of the generalized metaballs. We also present some extensions and the extended deformation model to include scale and rotation constraints. Experiments show that this deformation model is efficient and intuitive. It can deal with various constraints, which is difficult for traditional deformation model.
\end{abstract}

Keywords: Computer animation, Metaballs, Implicit surface, Constrained deformation.

\section{Introduction}

Efficient and intuitive methods for three-dimensional shape deformations play an important role in both geometric modeling and computer animation. Although the shape of an object can be finely controlled by interactively adjusting the positions of its vertices or control vertices, to most users, this manipulation is tedious and time-consuming. In this decade, two efficient techniques, namely physically based modeling and spatial deformation, have been proposed to solve the problem.

Physically based modeling technique produces very realistic deformations of the elastic objects by solving complex differential equations [1,2,3,4]. After the users specify the physical attributes (such as mass, friction, external forces, etc.) of the objects, the technique automatically generates the deformations and motions of the objects without any interaction. Although the technique is very powerful, it suffers from some drawbacks. Firstly, the technique involves a large amount of computation, it cannot be used as a real time interactive design tool. Secondly, the deformations produced by this technique are environment dependent. Finally, as there is no interaction during the simulation, it is very difficult to control the deformations of the objects. These disadvantages greatly limit the application of the technique in geometric modeling and computer animation.

The idea behind the spatial deformation techniques is to deform the whole space in which the objects are embedded instead of directly manipulating the vertices or control vertices of these objects. The first spatial deformation model was proposed by Barr [5]. According to Barr's method the transformation matrix is no longer constant but a function depending on the position of the individual points to which the transformation is applied. Obviously, Barr's model is a global approach hence difficult to deform the objects arbitrarily. The most popular spatial deformation technique is the free-form deformation (FFD) technique developed by Sederberg and Parry [6]. FFD is typically conducted by embedding an object to be deformed into a parametric space of a trivariate Bezier volume whose control points are organized as a lattice, the deformation of the object is obtained by moving the control points of the trivariate Bezier volumes. There have been many variant versions of FFD. Coquillart et al extended the FFD technique allowing composite lattices beside parallelepiped [7]. Similar techniques based on B-spline volumes or rational Bezier volumes were also proposed by other authors [8,9]. MacCraken et al developed a new FFD technique, in which the control points of the lattice can be arrayed in arbitrary topology [10]. Wyvill et al present a warping method for CSG/Implicit models [11].

Although FFD based methods can achieve a very variety of deformations, the user is forced to define some control points around the space to be deformed and then move these control points. This indirect interface may be 
unnatural for some applications. Hsu et al addressed this problem and proposed a direct interface that involves solving a complex equation system [12], but its cost is expensive. Borrel and Bechmann developed a general deformation model in which the deformation is defined by some user-specified point displacement constraints [13]. The desired deformation is obtained by selecting a solution obeying the constraints. Nevertheless, the shape of the resulting deformation in this method is not strongly correlated with the constraints except the constraints are satisfied. To overcome this problem, Borrel and Rappoport introduced a local deformation method which they term Simple Constrained Deformation (Scodef) [14]. In Scodef, the user defines some constraint points, each of which is associated with a user-defined displacement and an effective radius. The displacement of any point to be deformed is the blend of the local B-spline basis functions determined by these constraint points. Note that the deformation achieved by Scodef is both local and intuitive and the constrained points can be directly located on the boundary surface of the object to be deformed. To extend the flexibility of the local deformation, however, deformation models based on line, surface, and volume constraints are desired. Borrel and Rappoport pointed out that their model could not be generalized to deal with these kinds of constraints.

Motivated by the metaball technique, in this paper, we propose an innovative constrained deformation model based on the special potential function distribution of generalized metaballs. In our method, constraints are generalized to include point constraints, line constraints, surface constraints and volume constraints. The user need only define a set of constraints with desired displacements and an effective radius associated with each constraint. A generalized metaball is then set up at each constraint with a local potential function centered at the constraint falling to zero for points beyond the effective radius. The displacement of any point within the metaballs is a blend of these generalized metaballs. This deformation model produces a local deformation and is independent of representation of the underlying objects to be deformed. The constraints generate some "bumps" shapes over the space based on the type of constraint and its associated potential function, and they influence the final shape of the deformed object directly. The location and height of a bump are defined by a constraint and its influence space is determined by the constraint's effective radius. This method is very intuitive as the user can easily predict the deformed shape according to the constraints. For most constraints the computations required by the technique can be done very efficiently and the deformations can be implemented in real-time on current workstations.

\section{Constrained Local Deformation Based on Generalized Metaballs}

Metaball modeling is regarded as a flexible technique for implicit surface modeling. It is very convenient for designing closed surfaces and provides simple solutions for creating blends, ramifications and advanced human character design $[15,16,17,18,19,20,21]$. A good introduction of metaball modeling and implicit surface can be found in [22]. According to the basic formulation proposed by Blinn and Nishimura [15,16], a free-form surface is defined as an isosurface of a scalar field which is generated from some field generating points. The field value at any point is determined by the distance to the generating points. The parameters available for each metaball include the position of the generating point, the potential function etc.

Later Bloomenthal et al extended the original idea to include other complex sources such as lines, surfaces and volumes [20,21], which are termed as skeletons. The skeleton-based model provides an intuitive way to define the desired shapes with implicit surfaces. Let $C$ be the skeleton, $P(x, y, z)$ be a point in 3D space, $r(P, C)$ be the minimal distance from $P(x, y, z)$ to the individual points $Q(u, v, w)$ on the skeleton $C$ :

$$
r(P, C)=\inf _{Q \in C}\|P-Q\|
$$

Then the potential function associated with the skeleton $C$ can be defined as the composition of a potential function $f(r, R)$ which maps $\Re$ to $\Re$ and a distance function $r(P, C)$ which maps $\mathfrak{R}^{3}$ to $\mathfrak{R}[23]$ :

$$
F(r(P, C), R)=f(r, R) \circ r(P, C)
$$

where $R$ is a specified distance called effective radius. Euclidean space is often adopted as the distance space for calculating $r(P, C)$ and

$$
r(P, C)=\inf _{Q \in C} \sqrt{(x-u)^{2}+(y-v)^{2}+(z-w)^{2}}
$$

The field functions used for implicit surface modeling include Blinn's exponential function, Nishimura's piecewise quadric polynomial, Murakami's degree four polynomial and Wyvill's degree six polynomial[24]. In this paper, we adopt Wyvill's degree six polynomial as the finite potential function because this function blends well and can avoid the calculation of square root:

$$
f(r, R)=\left\{\begin{array}{rr}
-\frac{4}{9}\left(\frac{r}{R}\right)^{6}+\frac{17}{9}\left(\frac{r}{R}\right)^{4}-\frac{22}{9}\left(\frac{r}{R}\right)^{2}+1, & 0 \leq r \leq R \\
0 \quad & r>R
\end{array}\right.
$$

We extend the usage of metaball modeling to local space deformation. The field value of any point of an object is now defined as the weight of displacement from its original position. By interactively specifying the 
constraints and their effective radii, we can achieve various deformation effects. The constraints can either be points, lines, surfaces or volumes.

Let $C$ be a constraint skeleton, $R$ be the effective radius, and $S$ be the corresponding distance surface:

$$
S=\{P(x, y, z) \in S \mid r(P, C)=R\}
$$

We define tuple $M=\langle S, f(r, R)\rangle$ as a generalized metaball based on the skeleton $C$.

A general constrained deformation model based on generalized metaballs can then be defined as follows. Let $P=(x, y, z)$ be a point in $\mathfrak{R}^{3}, \operatorname{Deform}(P): \mathfrak{R}^{3} \rightarrow \mathfrak{R}^{3}$ be a deformation function which maps $P$ to $\operatorname{Deform}(P)$. Let $C_{i}$ be a constraint which is made up of points, lines, surfaces and volumes, $\Delta \mathbf{D}_{\mathbf{i}}$ be its displacement, $R_{i}$ be the effective radius of $C_{i}$. Then the deformation function effected by constraint $C_{i}$ is defined as:

$$
\operatorname{Deform}(P)=P+\Delta \mathbf{D}_{\mathbf{i}} F\left(r\left(P, C_{i}\right), R_{i}\right)
$$

Deformation model (5) has the following nice properties. For $\forall P \in C_{i}$, we have

$$
\operatorname{Deform}(P)=P+\Delta \mathbf{D}_{\mathbf{i}} F\left(0, R_{i}\right)=P+\Delta \mathbf{D}_{\mathbf{i}}
$$

If the distance from $P$ to constraint $C_{i}$ is lager than $R$, we have

$$
\operatorname{Deform}(P)=P+\Delta \mathbf{D}_{\mathbf{i}} F\left(R_{i}, R_{i}\right)=P
$$

Therefore, deformation function $\operatorname{Deform}(P)$ yields a local deformation which satisfies the constraint precisely in the constraint $C_{i}$, and does not affect the points outside the effective radius of the constraint.

The above model can be easily extended to deal with multiple constraints. The deformation function for $n$ constraints is defined as:

$$
\operatorname{Deform}(P)=P+\sum_{i=1}^{n} \Delta \mathbf{D}_{\mathbf{i}} F\left(r\left(P, C_{i}\right), R_{i}\right)
$$

The "bumps" generated by the constraints are blended by the potential function. By adjusting the constraints and their effective radii, the required deformation can be satisfied. Careful study shows that one constraint may sometimes impose deformation effect on other constraints although this does not prevent the application of the model. We say two constraints are disjoint if neither generalized metaball intersect the other constraint's skeleton. A set of constraints is disjoint if they are pairwise disjoint. Therefore for a disjoint set of constraints deformation model (8) can satisfy all the constraints. Model (8) has the following intuitive meaning: The displacement of point $P$ is the average of the displacements of the constraints weighted by their corresponding potential functions.

\section{The Computation of Generalized Metaballs}

From Formula (1) we can see that the key for calculating the deformation function lies in the computation of distance function $r\left(P, C_{i}\right)$. If $C_{i}$ is a point constraint, $r\left(P, C_{i}\right)$ is just the distance from point $P$ to $C_{i}$ i.e. $r\left(P, C_{i}\right)=\left\|P-C_{i}\right\|$. When $C_{i}$ is a line segment, a piece of surface, or a volume, the involved computations become complex. In the followings, we give the computation methods for some typical cases.

Line Segment Constraint: Let $C_{i}$ be a line segment determined by its end points $P_{0}=\left(x_{0}, y_{0}, z_{0}\right)$ and $P_{1}=\left(x_{1}, y_{1}, z_{1}\right)$, its length is $l$. We first transform this line segment into $\tilde{P}_{0} \tilde{P}_{1}$ on the $x$ axis by a transformation matrix $T$, where $\tilde{P}_{0}=(0,0,0) \quad \tilde{P}_{1}=(l, 0,0)$. For any point $P$ we apply the same transformation $T$ and obtain $\tilde{P}=(\tilde{x}, \tilde{y}, \tilde{z})$. As distance function is independent of the coordinate system,

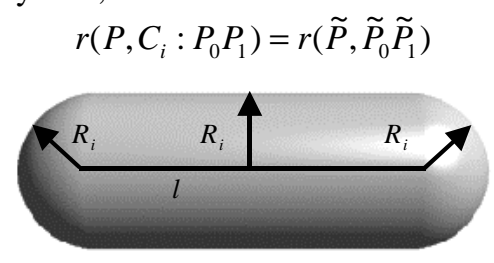

\section{Figure 1. The corresponding generalized metaball of a line segment}

That is, the distance from $P$ to $C_{i}$ is just the distance from $\tilde{P}$ to $\tilde{P}_{0} \tilde{P}_{1}$, therefore

$$
r\left(\tilde{P}, \tilde{P}_{0} \tilde{P}_{1}\right)=\left\{\begin{array}{lr}
\sqrt{\tilde{x}^{2}+\tilde{y}^{2}+\tilde{z}^{2}}, & \tilde{x}<0 \\
\sqrt{\tilde{y}^{2}+\tilde{z}^{2}}, & 0 \leq \tilde{x} \leq l \\
\sqrt{(\tilde{x}-l)^{2}+\tilde{y}^{2}+\tilde{z}^{2}}, & \tilde{x}>l
\end{array}\right.
$$

In order to reduce the computation time, $\tilde{P}_{0} \tilde{P}_{1}$ and transformation $T$ can be precomputed. As the potential function $f$ is a function of $r^{2}$, the square root computation can be eliminated by computing $r^{2}\left(\tilde{P}, \widetilde{P}_{0} \widetilde{P}_{1}\right)$ instead of $r$. The corresponding generalized metaball of a line segment is a cylinder with two hemispheres in both ends as illustrated in Figure 1. If the constraint $C_{i}$ is a line, the computation becomes much simpler as $r\left(P, C_{i}\right)$ is just the distance from $P$ to the line. The corresponding generalized metaball of a line is a cylinder whose radius and height are $R_{i}$ and $\infty$ respectively.

Polyline Constraint: Let constraint $C_{i}$ be a polyline defined by $P_{0} P_{1} P_{2} \cdots P_{n}$. For any line segment 
$P_{i-1} P_{i}(i=1,2, \cdots, n)$, we can obtain $r\left(P, P_{i-1} P_{i}\right)$ by the line segment constraint method as described above. The distance between any space point $P$ and $C_{i}$ is the minimum of the obtained distances:

$$
r\left(P, C_{i}: P_{0} P_{1} P_{2} \cdots P_{n}\right)=\min _{i \in[1, n]}\left\{r\left(P, P_{i-1} P_{i}\right)\right\}
$$

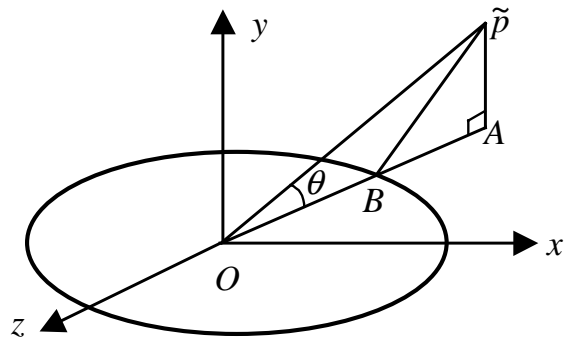

Figure 2. Distance calculation for a circle line

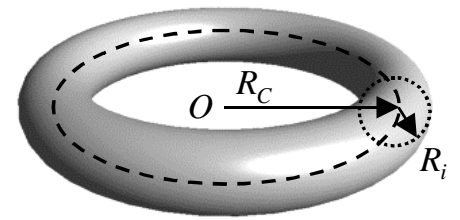

\section{Figure 3. Generalized metaball for a circle line}

Circle Line Constraint: Let $C_{i}$ be a circle line whose radius is $R_{C}$. We first transform the circle line onto the $x z$ plane by transformation matrix $T$, and its center is transformed into the origin. For any point $P$, we apply the same transformation matrix and obtain $\tilde{P}=(\tilde{x}, \tilde{y}, \tilde{z})$. From Figure 2 we know $O B=R_{C}, O \tilde{P}=\sqrt{\tilde{x}^{2}+\tilde{y}^{2}+\tilde{z}^{2}}$, thus

$$
r^{2}\left(P, C_{i}\right)=R_{C}{ }^{2}+\tilde{x}^{2}+\tilde{y}^{2}+\tilde{z}^{2}-2 R_{C} \sqrt{\tilde{x}^{2}+\tilde{z}^{2}}
$$

Its corresponding metaball is a torus whose major radius equals $R_{C}+R_{i}$ and minor radius equals $R_{C}-R_{i}$ as illustrated in Figure 3.

$n$ Degree Bezier Curve Constraint: Let $C_{i}$ be a Bezier curve $\mathbf{R}(u)$ of degree $n$. The minimal distance from a space point $P$ to $C_{i}$ either lies in its end points, or lies in the points satisfying the equation:

$$
(\mathbf{P}-\mathbf{R}(u)) \bullet \mathbf{R}_{\mathbf{u}}(u)=0
$$

This equation can be converted into a Bezier curve of degree $2 n-1$, then its roots can be solved by Bezier Clipping $[24,25,26]$. The value of the distance function is the minimum of them. It is easy to know the corresponding metaball is a generalized cylinder.

Disk Constraint: Let $C_{i}$ be a disk whose radius is $R_{C}$. We first calculate the distance $r_{1}$ from space point $P=(x, y, z)$ to the plane where the disk lies (See Figure 4). If the perpendicular point of $P$ lies within the disk, $r\left(P, C_{i}\right)=r_{1}$; otherwise we calculate the distance $r_{2}$ from
$P$ to the circle line, and set $r\left(P, C_{i}\right)=\min \left(r_{1}, r_{2}\right)$. The shape of the corresponding generalized metaball of a disk is shown in Figure 5.

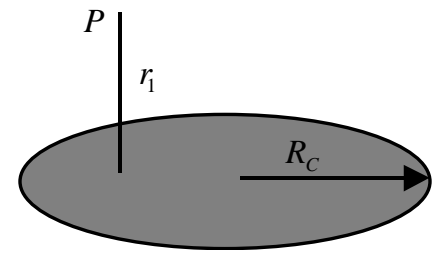

Figure 4. Disk constraint

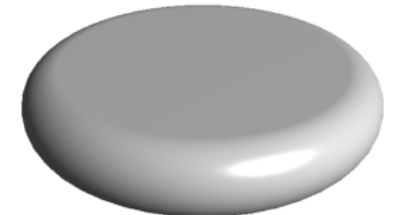

Figure 5. The generalized metaball for a disk

Planar Polygon Constraint: Let $C_{i}$ be a planar polygon $P_{0} P_{1} P_{2} \cdots P_{n} P_{0}$, the plane equation it lies is $A x+B y+C z+D=0$. Then the distance $r_{1}$ from a space point $P$ to the plane is

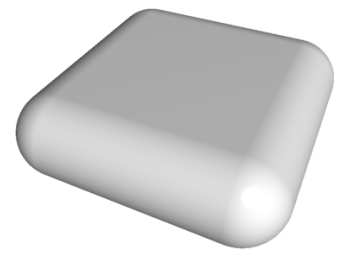

Figure 6. The generalized metaball for a square

$$
r_{1}=\frac{|A x+B y+C z+D|}{\sqrt{A^{2}+B^{2}+C^{2}}}
$$

If the perpendicular point of point $P$ lies in the polygon, $r\left(P, C_{i}\right)=r_{1}$; otherwise we calculate the distance $r_{2}$ from $P \quad$ to the polyline $P_{0} P_{1} P_{2} \cdots P_{n} P_{0}$, and set $r\left(P, C_{i}\right)=\min \left(r_{1}, r_{2}\right)$. Figure 6 shows the shape of the generalized metaball for a square.

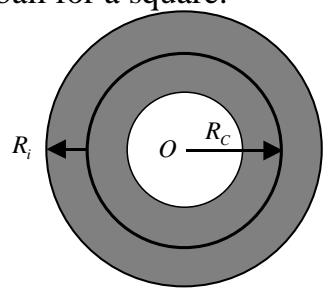

Figure 7. The generalized metaball for a sphere

Sphere Constraint: Let $C_{i}$ be a sphere whose radius is $R_{C}$, its center is $O\left(x_{C}, y_{C}, z_{C}\right)$. Then the distance from space point $P$ to the sphere is

$$
r\left(P, C_{i}\right)=\left|\sqrt{\left(x-x_{C}\right)^{2}+\left(y-y_{C}\right)^{2}+\left(z-z_{C}\right)^{2}}-R_{C}\right|
$$


The cross section for its corresponding metaball is shown in Figure 7.

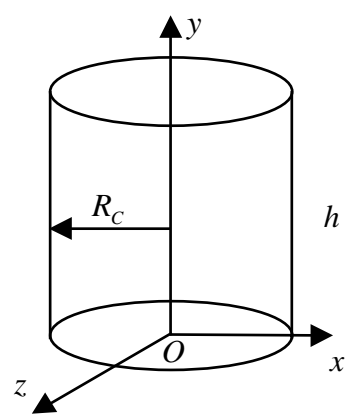

Figure 8. Cylinder constraint

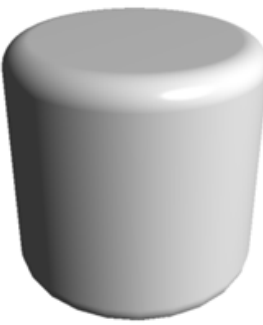

\section{Figure 9. The outer surface of the generalized} metaball for a cylinder constraint

Cylinder Constraint: Let $C_{i}$ be a cylinder whose radius is $R_{C}$ and whose height is $h$. We first transform the cylinder so that its bottom surface lies on the $x z$ plane and its center line coincides with $z$ axis (See Figure 8). By applying the transformation $T$ to the space point $P$ we obtain $\tilde{P}=(\tilde{x}, \tilde{y}, \tilde{z})$, then $r\left(P, C_{i}\right)$ can be easily determined. The shape of outer surface of the generalized metaball for a cylinder constraint is shown in Figure 9.

Sphere Volume Constraint: Let $C_{i}$ be a sphere volume, whose radius is $R_{C}$ and its center is $O\left(x_{C}, y_{C}, z_{C}\right)$. Obviously $r\left(P, C_{i}\right)$ equals 0 if a space point $P$ lies inside the sphere volume, otherwise the distance from $P$ to the sphere volume is:

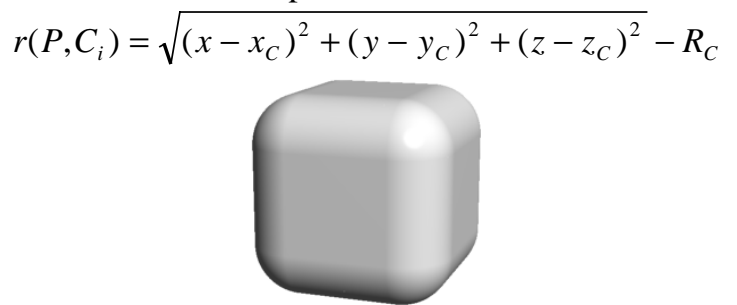

\section{Figure 10. Generalized metaball for a cube volume}

Cube Volume Constraint: Let $C_{i}$ be a cubic volume, whose edge length is $2 a$. We first apply transformation $T$ so that the center of the cube is located at the origin, and its edges are parallel to the three coordinate axes. After applying the same transformation $T$ to a space point $P$ we get $\quad \tilde{P}=(\tilde{x}, \tilde{y}, \tilde{z})$. If $\quad|\tilde{x}| \leq a \& \&|\tilde{y}| \leq a \& \&|\tilde{z}| \leq a$, $r\left(P, C_{i}\right)$ equals to 0 as $\tilde{p}$ lies in the cube. Otherwise the point nearest to $\tilde{p}$ either lies in the faces of the cube ( 6 cases ), or lies in the edges of the cube ( 12 cases), or lies in the vertices of the cube( 8 cases) according the position of the $\tilde{p}$. For each case the distance can be calculated easily. The shape of the generalized metaball for a cube voulme is shown in Figure 10.

For those constraints which are not listed above, their distance functions can be calculated similarly. When the deformation is applied to an object, the distance function $r\left(P, C_{i}\right)$ must be calculated for any vertex $P$ of the object, and thus the efficiency of the calculation of the distance function determines that of the algorithm. Note that the above deformation model is a local one. If the distance from a point on the candidate object to the constraint is larger than the effective radius of the constraint, this point is not affected. Thus we can adopt the bounding boxes or bounding spheres of the generalized metaballs to improve the efficiency of the algorithm. If a point does not lie in the bounding boxes of the generalized metaball of a constraint, this constraint has no effect to the point and hence its distance function calculation can be eliminated.

\section{Extensions}

In the local deformation model discussed above we adopt Wyvill's degree six polynomial as the potential function, this polynomial is in fact a special Bezier function. If we generalize the potential function to a Bezier function, more control freedoms can be obtained. The extended potential function can be rewritten as:

$$
f\left(r, R_{i}\right)=B e z_{i}(t)=\sum_{j=0}^{m} g_{j} B_{j}^{k}(t), \quad t \in[0,1]
$$

where $g_{0}$ is restricted to $1, g_{m}$ is restricted to 0 , $t=r / R_{i}, t=1$ if $r>R_{i}$. A user can use the remaining $m-1$ control points $\left\{g_{j}\right\}_{j=1}^{m-1}$ to control the distribution of the potential function. The purpose of the restrictions on $g_{0}$ and $g_{m}$ is to make metaballs blend well. Of course these restrictions can be removed if there is only one constraint or a user does not has the well-blend requirement. Moreover, we can deform both the constraint and its local area with distances less than $R_{U}$ to a userdefined displacement $\Delta D_{i}$ by adjusting the Bezier function as:

$$
f\left(r, R_{i}\right)=\left\{\begin{array}{cc}
1, & r \leq R_{U} \\
\operatorname{Bez}_{i}\left(\frac{r-R_{U}}{R-R_{U}}\right), & r>R_{U}
\end{array}\right.
$$


The shape of the reformed Bezier function is shown in Figure 11.

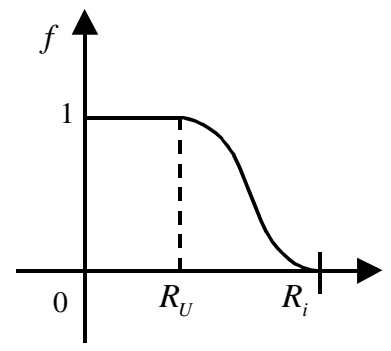

Figure 11. Reformed Bezier function

In the previous discussions the distance space we adopted is Euclidean distance, which is also known as spherical distance. The disadvantage of adopting such a distance is that the appearance of the resultant deformation is always of "bubble-shape". To weaken this drawback, other non-Euclidean metric spaces can be used to extend the variety of shapes of deformation. If we adopt n-norm metric space which is a straightforward generalization of the Euclidean distance,

$$
\begin{aligned}
r\left(P_{1}, P_{2}\right) & =\left\|\left(\left(x_{1}, y_{1}, z_{1}\right),\left(x_{2}, y_{2}, z_{2}\right)\right)\right\|_{n} \\
& =\left(\left|x_{2}-x_{1}\right|^{n}+\left|y_{2}-y_{1}\right|^{n}+\left|z_{2}-z_{1}\right|^{n}\right)^{1 / n}
\end{aligned}
$$

a lot of interesting results can be obtained. If $n$ equals 2 we obtain familiar Euclidean distance, the corresponding metaball for a point constraint is a sphere. If $n$ equals 1 ,

$$
\begin{aligned}
r\left(P_{1}, P_{2}\right) & =\left\|\left(\left(x_{1}, y_{1}, z_{1}\right),\left(x_{2}, y_{2}, z_{2}\right)\right)\right\|_{1} \\
& =\left(\left|x_{2}-x_{1}\right|+\left|y_{2}-y_{1}\right|+\left|z_{2}-z_{1}\right|\right)
\end{aligned}
$$

we obtain Manhattan distance, the corresponding metaball for a point constraint is a double pyramid. At the limit case $(n \rightarrow \infty)$,

$$
\begin{aligned}
r\left(P_{1}, P_{2}\right) & =\left\|\left(\left(x_{1}, y_{1}, z_{1}\right),\left(x_{2}, y_{2}, z_{2}\right)\right)\right\|_{\infty} \\
& =\max \left(\left|x_{2}-x_{1}\right|,\left|y_{2}-y_{1}\right|,\left|z_{2}-z_{1}\right|\right)
\end{aligned}
$$

One obtains city block distance, the corresponding metaball for a point constraint is a cube. By adopting different metric space, the influence range can be quite different. Therefore we can take $n$ as an animatable parameter to adjust the influence range of a constraint. But adopting $n$ as an animatable parameter usually requires some costly computations in $r\left(P_{1}, P_{2}\right)$, an alternative way is to linearly interpolate the Euclidean distance, the Manhattan distance and the city block distance to calculate other form distance in metric space. For example, if we calculate the distance in the following way:

$(1-u)\left\|\left(\left(x_{1}, y_{1}, z_{1}\right),\left(x_{2}, y_{2}, z_{2}\right)\right)\right\|_{2}+u\left\|\left(\left(x_{1}, y_{1}, z_{1}\right),\left(x_{2}, y_{2}, z_{2}\right)\right)\right\|_{\infty}$

Then, by animating parameter $u$ from 0 to 1 , the influence range will change from a sphere to a cube, but the involved computation is quite small. Blanc even presented some anisotropy distance functions such as axial distance function and radial distance function to control precisely the shape of the resulting soft object [23]. Introducing them into our deformation model can bring even more precise control of the influence range.

In the previous discussion, each space coordinate is treated symmetrically. To accommodate even finer control of the influence range, we can treat each space coordinate differently so as to provide asymmetric, nonisotropic space deformation around the constraints. For example, let $R_{i x}, R_{i y}, R_{i z}$ be the effective radii for $x, y, z$ axes respectively, $\quad C_{i}=\left(C_{i x}, C_{i y}, C_{i z}\right), \quad P=(x, y, z), \quad$ by redefining $r / R_{i}$ in formula (3) as:

$$
\sqrt{\frac{\left(x-C_{i x}\right)^{2}}{R_{i x}{ }^{2}}+\frac{\left(y-C_{i y}\right)^{2}}{R_{i y}{ }^{2}}+\frac{\left(z-C_{i z}\right)^{2}}{R_{i z}{ }^{2}}}
$$

We can achieve asymmetric space deformation.

\section{Deformation by Local Rotation and Scale}

In section 3, we discussed the shape deformation by local displacement or translation. A natural extension is to generalize the deformation model so that it can deal with local rotation and scale. Both of these kinds of deformations are of important use in computer animation.

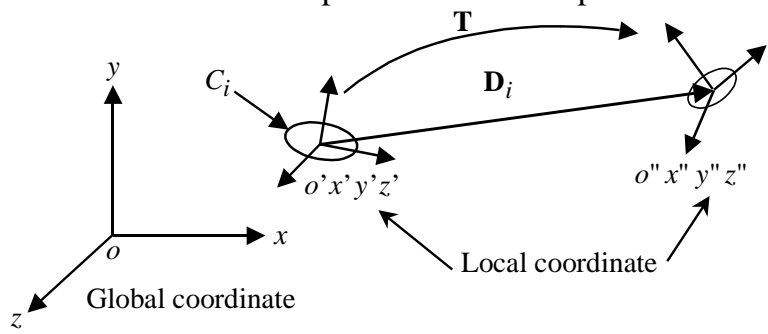

\section{Figure 12. Local coordinate system for a constraint}

An intuitive interface can be set up by attaching a local coordinate system to each constraint $C_{i}$ as illustrated in Figure 12. Let the local coordinate system at $C_{i}$ be $o^{\prime} x^{\prime} y^{\prime} z^{\prime}$, then a user moves, rotates and scales the coordinate system until all the translation, rotation and scale requirements are satisfied. Let the destination coordinate system be $o^{\prime \prime} x^{\prime \prime} y^{\prime \prime} z^{\prime \prime}$ and the transformation matrix from source coordinate system $o^{\prime} x^{\prime} y^{\prime} z^{\prime}$ to the destination coordinate system $o^{\prime \prime} x^{\prime \prime} y^{\prime \prime} z^{\prime \prime}$ be $\mathbf{M}$. $\mathbf{M}$ is made up of translation matrix $\mathbf{T}\left(D_{x}, D_{y}, D_{z}\right)$, scale matrix $\mathbf{S}\left(S_{x}, S_{y}, S_{z}\right)$ and rotation matrix $\mathbf{R}\left(\theta_{x}, \theta_{y}, \theta_{z}\right)$, i.e.,

$$
\mathbf{M}=\mathbf{T}\left(D_{x}, D_{y}, D_{z}\right) \mathbf{S}\left(S_{x}, S_{y}, S_{z}\right) \mathbf{R}\left(G_{x}, \theta_{y}, G_{z}\right)
$$

For any space point $P$ to be deformed, we first transform it into the local coordinate system $o^{\prime} x^{\prime} y^{\prime} z^{\prime}$ and obtain $P^{\prime}$, then multiply $P^{\prime}$ with transformation matrix $\hat{\mathbf{M}}$ and

$$
\hat{\mathbf{M}}=\mathbf{T}\left(\hat{D}_{x}, \hat{D}_{y}, \hat{D}_{z}\right) \mathbf{S}\left(\hat{S}_{x}, \hat{S}_{y}, \hat{S}_{z}\right) \mathbf{R}\left(\hat{\theta}_{x}, \hat{\theta}_{y}, \hat{\theta}_{z}\right)
$$


where

$$
\begin{aligned}
\left(\hat{D}_{x}, \hat{D}_{y}, \hat{D}_{z}\right) & =F\left(r\left(P, C_{i}\right), R_{i}\right)\left(D_{x}, D_{y}, D_{z}\right), \\
\left(\hat{\theta}_{x}, \hat{\theta}_{y}, \hat{\theta}_{z}\right) & =F\left(r\left(P, C_{i}\right), R_{i}\right)\left(\theta_{x}, \theta_{y}, \theta_{z}\right), \\
\left(\hat{S}_{x}, \hat{S}_{y}, \hat{S}_{z}\right)=(1,1,1) & +F\left(r\left(P, C_{i}\right), R_{i}\right)\left(S_{x}-1, S_{y}-1, S_{z}-1\right)
\end{aligned}
$$

Suppose the obtained point be $P^{\prime \prime}$. Finally we transform $P^{\prime \prime}$ back to the global coordinate system and obtained the deformed image of point $P$. If there is only translation, i.e. $\left(\hat{\theta}_{x}, \hat{\theta}_{y}, \hat{\theta}_{z}\right)=0$ and $\left(\hat{S}_{x}, \hat{S}_{y}, \widetilde{S}_{z}\right)=(1,1,1)$, the result is the same as the deformation model discussed in section 3 .

In the previous discussion, we use Euler angles $\left(\hat{\theta}_{x}, \hat{\theta}_{y}, \hat{\theta}_{z}\right)$ to describe the rotation of the coordinate system. However, Euler angle representation suffers from several disadvantages [27]. Firstly, Euler rotations must be applied in a particular order because they do not commute. Secondly, it suffers from non-uniformity. A fixed change in Euler angles does not always yield the same amount of rotation change. Thirdly, Euler angle representation suffers from "gimbal lock". An alternative is to use quaternion instead of Euler angles [27]. Euler angles $\left(\hat{\theta}_{x}, \hat{\theta}_{y}, \hat{\theta}_{z}\right)$ can be converted into a quaternion

$$
q=\left(\cos \frac{\theta}{2}, \sin \frac{\theta}{2} \mathbf{n}\right)=(w, x, y, z)
$$

where $\theta$ is the rotation angle and $\mathbf{n}$ is the unit rotation axis. By converting quaternion $q$ into rotation matrix $\mathbf{R}(\theta)$, we get

$$
\mathbf{R}(\theta)=\left[\begin{array}{ccc}
1-2 y^{2}-2 z^{2} & 2 x y-2 w z & 2 x z+2 w y \\
2 x y+2 w z & 1-2 x^{2}-2 z^{2} & 2 y z-2 w x \\
2 x z-2 w y & 2 y z+2 w x & 1-2 x^{2}-2 y^{2}
\end{array}\right]
$$

In this case, the transformation matrix $\hat{\mathbf{M}}$ is refined as:

$$
\hat{\mathbf{M}}=\mathbf{T}\left(\hat{D}_{x}, \hat{D}_{y}, \hat{D}_{z}\right) \mathbf{S}\left(\hat{S}_{x}, \hat{S}_{y}, \hat{S}_{z}\right) \hat{\mathbf{R}}(\hat{\theta})
$$

where

$$
\hat{\theta}=\theta F\left(r\left(P, C_{i}\right), R_{i}\right) .
$$

As quaternions interpolate only one angle instead of three Euler angles, it can generate smoother rotation interpolation and hence more fluid deformation. Given a set of quaternions, they can be spherical interpolated using a general construction scheme [28].

\section{The Animation of the Deformations}

The above deformation model can be conveniently applied to generate a deformation animation. We present two ways to simulate the deformation process of an object. The first is to apply a set of the different constraints to the same object to obtain a sequence of deformed objects. Since these objects possess the same number of vertices and the same topology, we can blend them to generate the intermediate shape by interpolating the corresponding vertices. The other method is to interpolate the corresponding parameters of the keyframe constraints to generate the intermediate constraints, the intermediate constraints are then applied to produce the deformation of the object for the intermediate frames. In fact, a constraint can be completely determined by parameter set $\Omega$ :

$$
\Omega=\left\{C_{i}, \Delta \mathbf{D}_{i}, S_{x}, S_{y}, S_{z}, \theta, R_{i x}, R_{i y}, R_{i z}, g_{1}, g_{2}, \cdots, g_{m-1}\right\}
$$

Given the parameter set of the keyframes, traditional parametric key frame techniques can be used to generate the intermediate parameter set of the constraint.

Since both methods are based on parametric key frame techniques, which are provided by many animation systems, our deformation animation model can be conveniently incorporated into these animation systems.

\section{Experiments}

We implemented our algorithm on a SGI Indy Workstation. Figure 13 shows the potential function distribution of the line constraint, disk constraint, square constraint, polyline constraint, point constraint adopting Euclidean distance and point constraint adopting Mahattan distance respectively, all of them are obtained by applying corresponding constraints to a grid. Figure 14 is the wireframe of an undeformed cow, and Figure 15 is the deformed cow by locating a line constraint on its back. Figure 16(a) is an undeformed teapot, Figure 16(b) is the deformed teapot by applying two plane constraints, one is put on its top and the other is put on its left. Figure 17 shows an undeformed cow and a plane constraint, the constraint is put on the right of the cow with a $45^{0}$ to the $x y$ plane where the cow lies. Figure 18 shows the animation sequence obtained by animating the displacement of a plane constraint. From the two examples we can see that a plane constraint is like a magnet, it attracts the points within the influence range. Figure 18 shows a "Z" deformed from a grid, there are 12 point constraints corresponding to 12 metaballs in the environment. Please compare this deformation with that in Figure 13(d). Figure 20 shows the undeformed cow and a sphere volume constraint, the small sphere is the constraint, the big sphere is the generalized metaball indicating the influence range of the constraint and the line shows the displacement $\Delta \mathbf{D}$. The 3D-morphing sequence in Figure 21 is achieved by animating $\Delta \mathbf{D}$ of the sphere volume constraint. We note that only the vertices of the cow which lie within the big sphere are deformed and other vertices are not affected at all. Figure 22 shows the animation sequence by animating the scale constraint of a sphere volume constraint, all the points in the head of the cow satisfy the constraint. The 3Dmorphing sequence in Figure 23 is achieved by animating the rotation of the sphere volume constraint, the rotation 
constraint $\theta$ is $-120^{\circ}$ around axis $(\sqrt{2} / 2, \sqrt{2} / 2,0)$. As the whole head of the cow is within the sphere volume, all the points on the head rotate $-120^{\circ}$ and hence the head keeps the same shape.

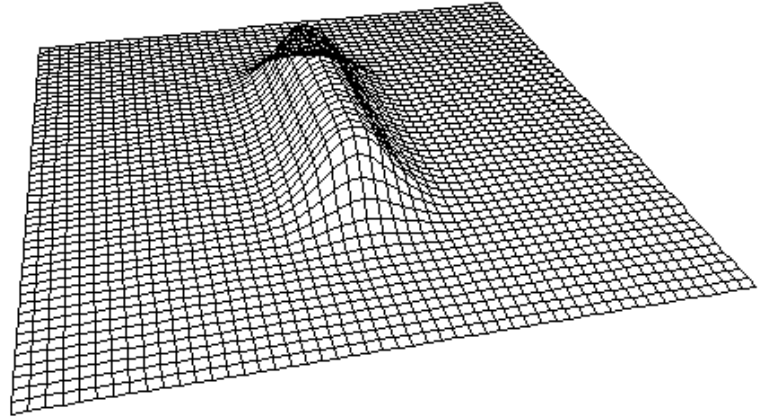

(a) Line segment constraint

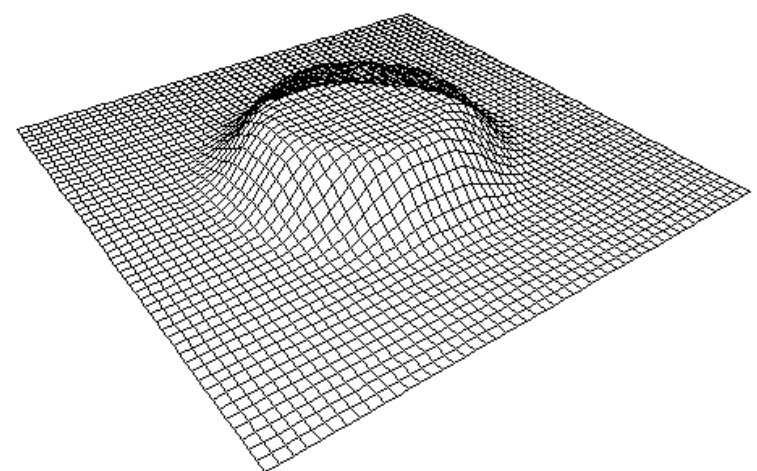

(b) Disk constraint

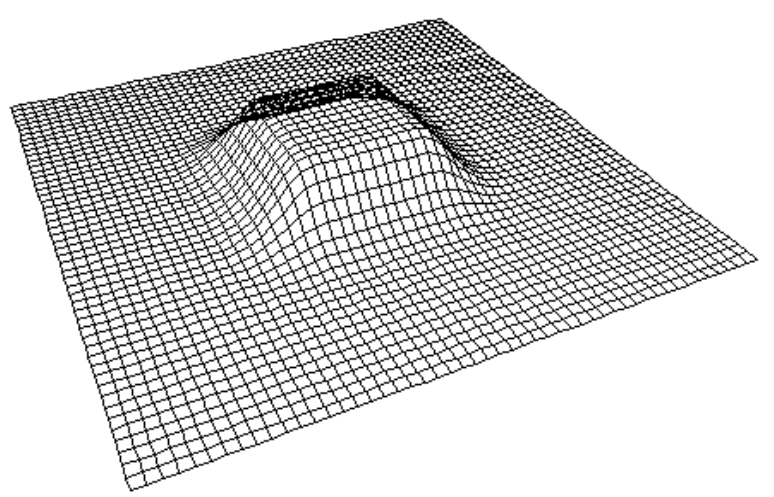

(c) Square constraint

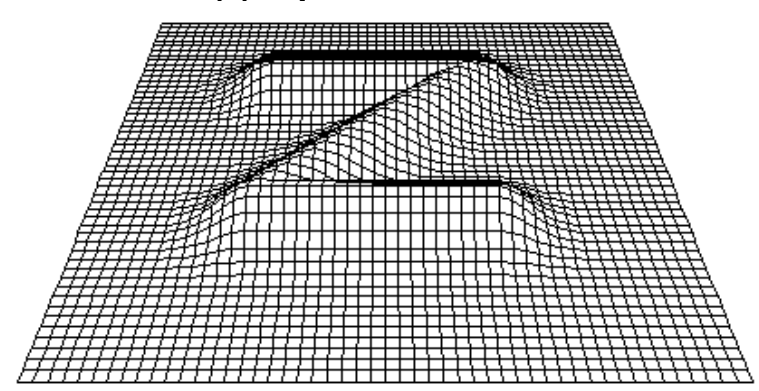

(d) Polyline constraint

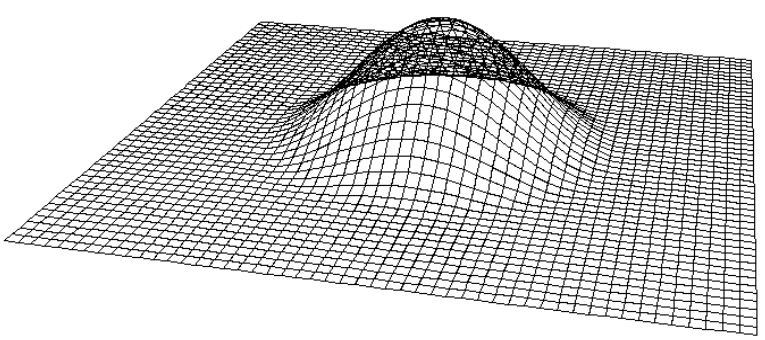

(e) Point constraint adopting Euclidean distance

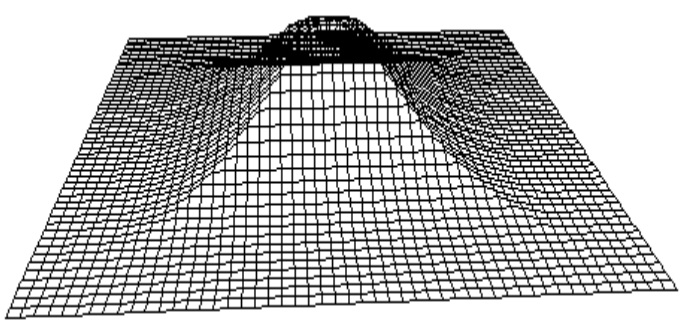

(f) Point constraint adopting Manhattan distance Figure 13. The potential function distribution for some constraints

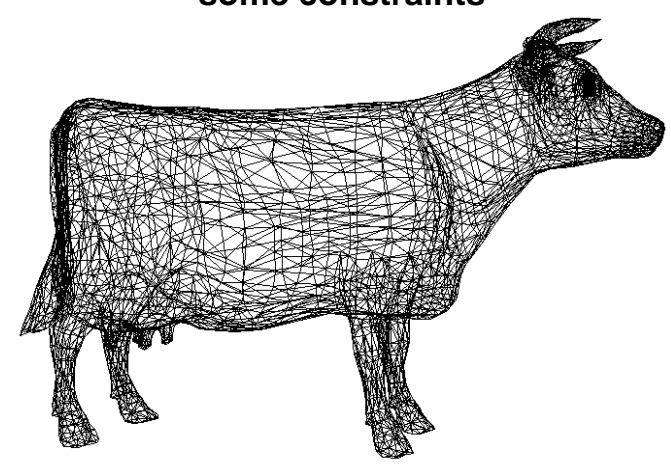

Figure 14. The wireframe of an undeformed cow

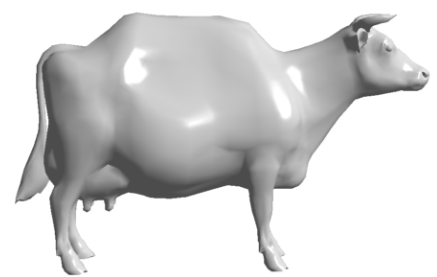

Figure 15. Deformed cow by a line constraint

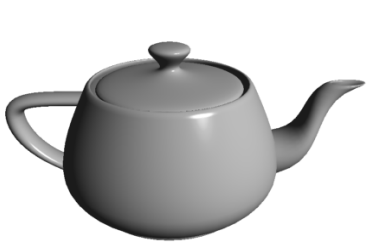

(a) Before deformation

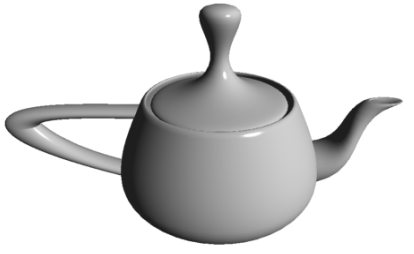

(b) Deformed teapot by two plane constraints 


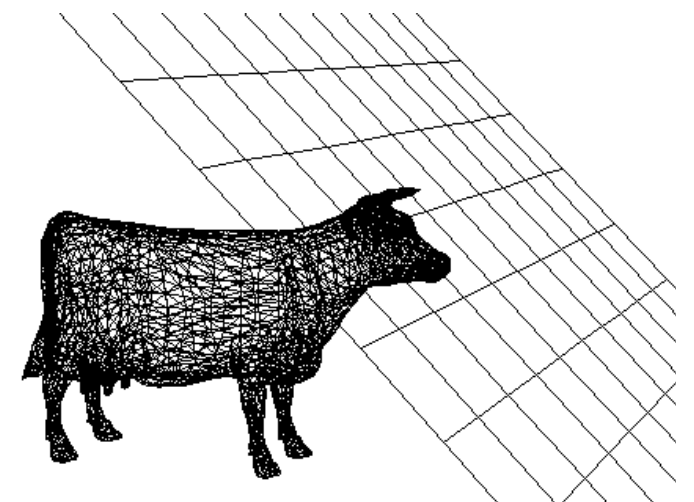

Figure 17. Undeformed cow and a plane constraint
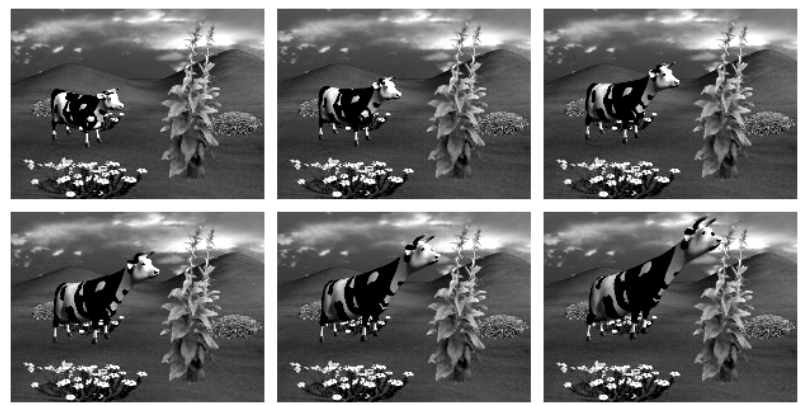

Figure 18. 3D-morphing sequence by animating the displacement of a plane constraint

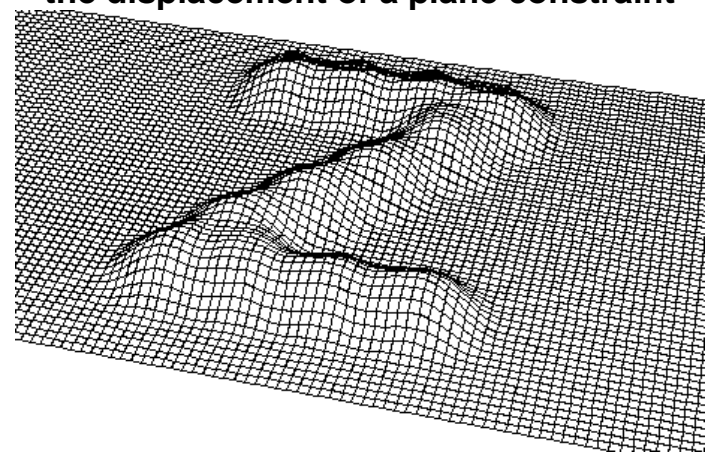

Figure 19. " $Z$ " deformed from a plane

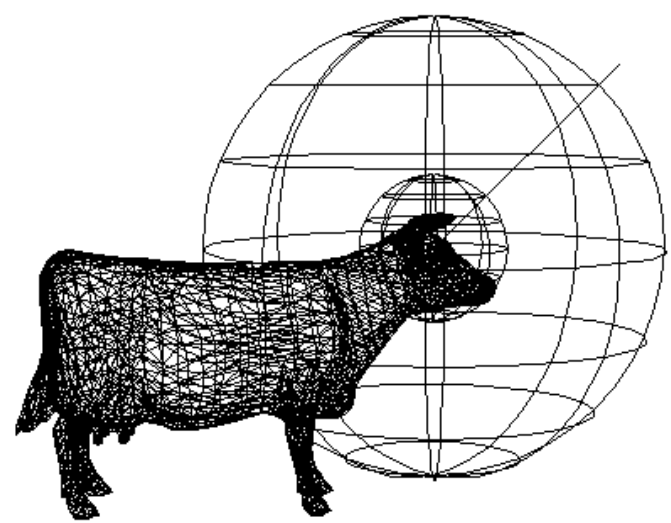

Figure 20. Undeformed cow and a sphere volume constraint
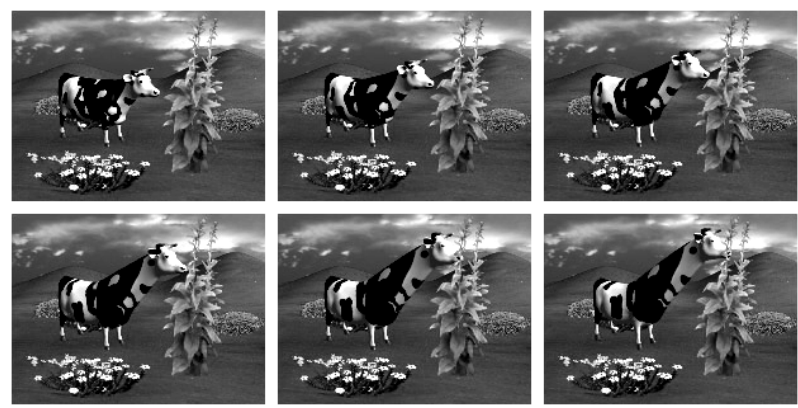

Figure 21. 3D-morphing by animating the displacement of a sphere volume constraint
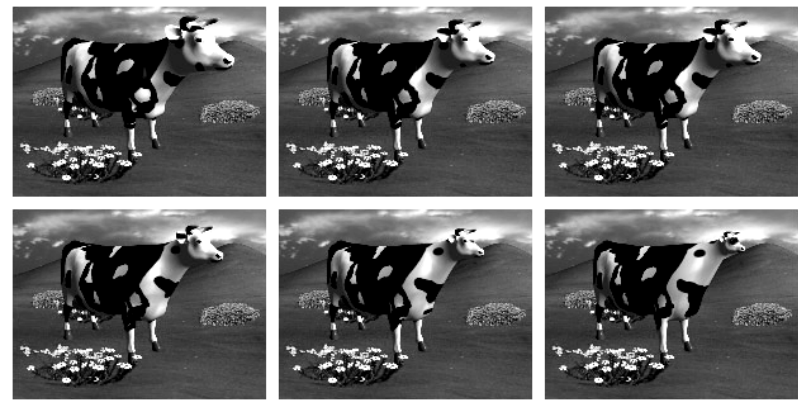

Figure 22. 3D-morphing by animating the scale of a sphere volume constraint
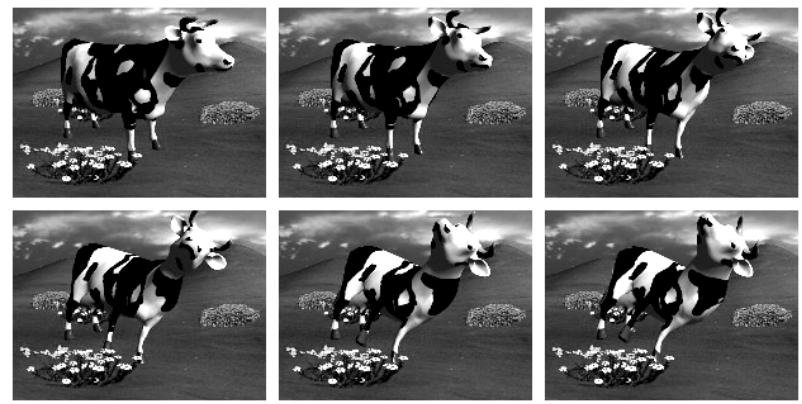

Figure 23. 3D-morphing by animating the rotation of a sphere volume constraint

\section{Conclusions}

A general constrained deformation model is presented in this paper. After a user specifies a series of constraints which can be made up of points, lines, surfaces and volumes, their effective radii and maximum displacements, the deformation model creates a set of generalized metaballs taking the constraints as the skeletons. Each metaball determines a local influence region and is associated with a local potential function. The potential function centered at the constraint and falling to zero for points beyond the effective radius. We present methods for calculating the distance functions for some typical constraints such as point, line segment, disk, Bezier curve, polygon, sphere volume etc. One advantage of our deformation model is that it is independent of the 
representation of the underlying objects and can apply to both polygon mesh and parametric surfaces. For most of the useful constraints, the algorithm is of high efficiency because the calculation involved is simple, and can be implemented interactively in current workstations. Compared with other deformation method, this deformation model has following features: (1). Generality. This method can not only deal with point constraint but also line, surface and volume constraints, which are difficult for traditional methods. The scale constraint and rotation constraint can be dealt with too in a systematic way. (2). Intuition. for a specified constraint, a user can easily imagine the deformation effects aroused by the constraint. (3) Locality. Only points located in the local influence range are affected, therefore it provides a useful tool for local shape adjustment. (4). Compatibility. The deformation model can be easily incorporated into most existing animation systems.

Acknowledgements The authors are grateful to Dr. Jieqing Feng, Prof. Hujun Bao and the anonymous reviewers for their constructive suggestions. This work is supported by National Natural Science Foundation of China and Natural Science Foundation of Zhejiang Province.

\section{References}

[1] D. Terzopoulos, J. Platt, A. H. Barr and K. Fleischer, "Elastically Deformation Models", Computer Graphics, Vol. 21, No. 4, 1988, pp. 205-214

[2] J. Platt, and A. H. Barr, "Constraints Methods for Flexible Models”, Computer Graphics, Vol.22, No.4, 1988, pp.279288.

[3] D. Terzopoulos, and K. Fleischer, "Modeling Inelastic Deformation: Viscoelasticity, Plasticity, Fracture", Computer Graphics, Vol. 22, No.4, 1988, pp.269-278.

[4] D. Terzopoulos, and A. Witkin, "Physically-based Methods with Rigid and Deformable Components", IEEE Computer Graphics \& Applications, Vol. 8, 1988, pp.41-51.

[5] A. H. Barr, "Global and Local Deformation of Solid Primitives", Computer Graphics, Vol. 18, No.3, 1984, pp. 21-30.

[6] T. W. Sederberg, and S. R. Parry, "Free-Form Deformation of Solid Geometric Models", Computer Graphics, Vol. 20, No.4, 1986, pp. 537-541.

[7] S. Coquillart, "Extended Free-Form Deformation: A Sculpturing Tool for 3D Geometric Modeling", Computer Graphics, Vol. 24, No.4, 1990, pp.187-193.

[8] P. Kalar, A. Mangli, M. Thalmann and D. Thalmann, "Simulation of Facial Muscle Actions Based on Rational Free-Form Deformations", Computer Graphic Forum, Vol. 11, 1992, pp. 59-69.

[9] H. J. Lamousin, W. N., Waggenspack, "NURBS-based Free-form Deformations", IEEE Computer Graphics \& Applications, Vol.14, No.9, 1994, pp. 59-65.
[10] R. MacCracken and K. I. Joy, "Free-Form Deformations with Lattices of Arbitrary Topology", Computer Graphics, Vol. 26, No.3, 1996, pp. 181-188.

[11] B. Wyvill and K. V. Overveld, "Warping as a Modelling Tool for CSG/Implicit Models", Proc. Of Shape Modeling International'97, University of Aizu, Japan, IEEE Computer Society Press, 1997, pp. 205-214.

[12] W. Hsu, J. Hughes, and H. Kaufmann, "Direct Manipulations of Free-Form Deformations", Computer Graphics, Vol. 26, No.2, 1992, pp. 177-184.

[13] P. Borrel, D. Bechmann, "Deformation of N-dimensional Objects", International Journal of Computational Geometry \& Applications, Vol.1 No.4, 1991, pp.427-453.

[14] P. Borrel, A. Rappoport, "Simple Constrained Deformations for Geometric Modeling and Interactive Design”, ACM Transactions on Graphics, Vol.13, No.2, 1994, pp.137-155.

[15] J. F. Blinn, "A Generalization of Algebraic Surface Drawing", ACM Transactions on Graphics, Vol. 1, No.3, 1982, pp. 235-256.

[16] H. Nishimura, M. Hirai, and T. Kawai, "Object Modeling by Distribution Function and a Method of Image Generation", Transactions on IECE, Vol.68-D, No.4, 1985, pp.718 725,

[17] G. Wyvill, C. McPheeters and B. Wyvill, "Data Structure for Soft Objects", The Visual Computer, Vol.2, 1986, pp.227-234

[18] B. Wyvill, G. Wyvill, "Field Functions for Implicit Surfaces", The Visual Computer, Vol. 5, 1989, pp.75 82.

[19] J. Shen, D. Thalmann, "Interactive Shape Design Using Metaballs and Splines", Proc. of Implicit Surfaces'95, Edited by Brian Wyvill and Marie-Paule Gascuel, Grenoble, France, 1995, pp.187-196.

[20] J. Bloomenthal, B. Wyvill, "Interactive Techniques for Implicit Modeling", Computer Graphics, Vol.24, No.2, 1990, pp.109 116.

[21] J. Bloomenthal, and K. Shoemake, "Convolution Surfaces", Computer Graphics, Vol.25, No.4, 1991, pp.251-256.

[22] J. Bloomenthal, C. Bajaj, J. Blinn, M. Cani-Gascuel, A. Rockwood, B. Wyvill, and G. Wyvill, An Introduction to Implicit Surfaces, Morgan Kaufmann Publishers, 1997.

[23] C. Blanc and C. Schlick, "Extended Field Functions for Soft Objects", Implicit Surfaces'95 Workshop, Editors: M.P. Gascuel \& B. Wyvill, 1995, pp. 21-32.

[24] T. Nishita and E. Nakamae, "A Method for Displaying Metaballs by Using Bezier Clipping”, Computer Graphics Forum, Vol.13, No.3, 1994, pp.271-280.

[25] P. J. Schneider, "Solving the Nearest-point-on-curve Problem", in Graphics Gems I, A.S. Glassner(Ed), Academic Press, 1990, pp.607-611.

[26] J. C. Hart, "Sphere Tracing: A Geometric Method for the Antialiased Ray Tracing of Implicit Surfaces", The Visual Computer, Vol.12, 1996, pp.527-545.

[27] K. Shoemake, "Animating Rotation with Quaternion Curves", Computer Graphics, Vol.19, No.3, 1985, pp.245254.

[28]M. J. Kim, M.S. Kim and S.Y. Shin, "A General Construction Scheme for Unit Quaternion Curves with Simple High Order Derivatives", Computer Graphics, Vol.29, No.3, 1995, pp.369-376. 